\title{
Relief of Secondary Headaches with High Thoracic Erector Spinae Plane Block
}

This article was published in the following Dove Press journal:

Local and Regional Anesthesia

\author{
Nadia Hernandez' \\ Grace Guvernator' \\ George Ansoanuur (1D ${ }^{2}$ \\ Michelle $\mathrm{Ge}^{3}$ \\ Precious Tabansi' \\ Thanh-Thuy Le' \\ Salameh S Obeidat ${ }^{4}$ \\ Johanna de Haan' \\ 'Department of Anesthesiology, Division \\ of Regional Anesthesiology and Acute \\ Pain Medicine, University of Texas Health \\ Science Center at Houston, McGovern \\ Medical School, Houston, TX 77030, \\ USA; ${ }^{2}$ Department of Neurology, \\ Division of Trauma, Concussion and \\ Sports Neuromedicine, University of \\ Florida College of Medicine, Gainesville, \\ FL 32610, USA; ${ }^{3}$ Department of \\ Anesthesiology, Division of Chronic Pain \\ Management, McGovern Medical School, \\ Houston, TX 77030, USA; ${ }^{4}$ Department \\ of Anesthesiology, Beth-Israel \\ Deaconness Medical Center, Harvard \\ Medical School, Boston, MA, USA
}

Correspondence: Johanna de Haan Tel +l 713-500-6200

Email johanna.b.dehaan@uth.tmc.edu

\begin{abstract}
Intractable headaches can be debilitating, often leading to significant distress, prolonged medical treatment, and unanticipated hospital admissions. There have been significant advances in the treatment of primary intractable headaches such as migraines, tension headaches, and cluster headaches beyond medical management. Treatments may now include interventional strategies such as trigger-point injections, peripheral nerve stimulators, or peripheral nerve and ganglion blocks. There are few studies, however, describing the use of interventional techniques for the management of intractable secondary headaches, including those attributed to injury or infection. A new regional anesthetic technique, the erector spinae plane (ESP) block, was initially used for neuropathic thoracic pain. ESP block has since been reported to provide acute and chronic pain relief of the shoulder, spine, abdomen, pelvis, thorax, and lower extremity. Additionally, there has been one case report to describe the use of the ESP block in the treatment of refractory tension headache. We report four cases of effective analgesia for intractable secondary headache resistant to medical management with high thoracic ESP blocks. In each case, the ESP block provided instant pain relief. We suggest that the findings of this case series indicate that the ESP block may be a useful intervention in patients with severe secondary headache or posterior cervical pain where conventional therapies have limited success, though more studies are necessary.
\end{abstract}

Keywords: erector spinae plane block, refractory headache, head pain, neck pain, scalp pain, craniotomy pain

\section{Introduction}

Refractory headache is a term used to describe several headache subtypes that are defined as being resistant to treatment or are intractable. ${ }^{1}$ Common refractory headache subtypes include primary headaches such as migraine, tension, and cluster headache, as well as secondary headaches attributable to trauma, inflammation, and infection. $^{2}$ Refractory secondary headaches can be debilitating, leading to unanticipated emergency room visits and hospital admissions. Pharmacologic treatment of secondary headache has several limitations due to drug interactions, contraindications, allergies, and intolerable side effects. Regional anesthesia has been shown to provide prompt, complete, and prolonged relief of refractory primary headaches and their associated autonomic symptoms. ${ }^{3,4}$ However, there is a paucity of evidence for the use of peripheral nerve blocks in the treatment of intractable secondary headache, except for greater occipital nerve block for postdural puncture headache (PDPH) and cervicogenic headache. ${ }^{5-8}$ Greater occipital (GON), lesser occipital (LON), and supraorbital nerve (SON) blocks, as well as stellate and sphenopalatine 
ganglion (SPG) blocks, have successfully treated secondary headaches, although this literature is limited to retrospective studies, small case series, and case reports.

A recently described fascial plane block that has successfully terminated primary headaches is the erector spinae plane (ESP) block. The ESP block was first reported in 2016 by Forero et al as an ultrasound-guided fascial plane block used to treat chronic thoracic pain. ${ }^{9}$ Local anesthetic injected in the plane between the transverse process (TP) and the erector spinae muscles spreads in a craniocaudal direction, anesthetizing the dorsal and ventral rami of adjacent spinal nerves. Twenty milliliters $(\mathrm{mL})$ injection of $0.5 \%$ ropivacaine over the fifth TP resulted in analgesia of intercostal nerves 3-9. Since then, the ESP block has been reported at many different spinal levels and has been used to provide acute post-surgical pain relief for cervical spine, thoracic spine, lumbar spine, shoulder, abdomen, pelvis, thorax, and lower extremity surgeries. ${ }^{10-16}$ A report by Ueshima et al noted relief of tension headache in two patients following the use of T4 ESP block. ${ }^{17}$ In this manuscript, we report four cases in which bilateral T4 ESP blocks provided significant relief of headaches secondary to various pathologies.

\section{Methods}

Procedural consent and pre-procedure pain scores were obtained using the numerical pain scale (NPS) by the acute pain service. Patients were transferred to the block room, connected to pulse oximetry, non-invasive blood pressure and continuous electrocardiographic monitors and positioned in left lateral decubitus. A high-frequency linear or lowfrequency curvilinear transducer (SonoSite, Bothell, WA) was placed in a sagittal plane over the 4th TP. The TP, pleura and erector spinae muscles were identified on ultrasound, as in Figure 1. After skin sterilization, a 22-gauge blunt-tipped echogenic needle (BBraun, Bethlehem, PA) was advanced in-plane from caudal to cranial until the needle made contact with the T4 TP. Normal saline was used to confirm needle tip location in the plane between the erector spinae muscles and TP. Twenty $\mathrm{mL}$ of $0.25 \%$ bupivacaine with $2-4 \mathrm{mg}$ of preservative-free dexamethasone was injected into the plane bilaterally, aspirating every $5 \mathrm{~mL}$ to ensure that the needle tip had not been placed intravascularly or intrapleural. Patients were monitored for 30-60 mins after the procedure. All patients provided written informed consent for publication of details regarding their case, including publication of images. The institutional research ethics committees at both our affiliated hospital, the Clinical Innovation and Research

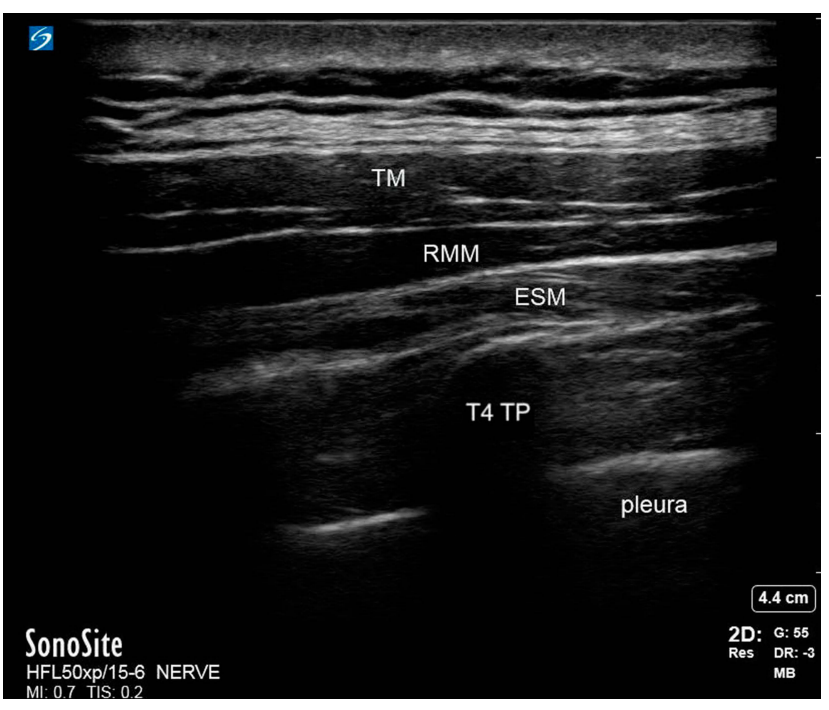

Figure I Patient ultrasound.

Abbreviations: TM, trapezius muscle; RMM, rhomboid major muscle; ESM, erector spinae muscle; T4 TP, thoracic 4 transverse process.

Institute of Memorial Hermann Hospital, and our university, the Center for the Protection of Human Subjects at UTHealth Houston, have reviewed and approved of this study.

\section{Case I - Meningitic Cervical Pain and Headache}

A 29-year-old female presented with signs and symptoms of acute meningitis, notably severe headache with nuchal pain and rigidity. Lumbar puncture was performed by the neurology service with a 3.5 -inch 22-gauge needle at the L2-L3 spinal interspace and cerebrospinal fluid (CSF) was obtained. Opening pressure was $18 \mathrm{~mm} \mathrm{H}_{2} \mathrm{O}$, closing pressure $12 \mathrm{~mm} \mathrm{H}_{2} \mathrm{O}$, with a total of $12 \mathrm{~mL}$ of clear CSF drained. Results from the fluid analysis showed Herpes Simplex Virus 2 (HSV2) meningitis without encephalitis. The patient received a course of intravenous acyclovir and transitioned to an oral antiviral regimen, but was unable to be discharged due to severe uncontrolled headache and neck pain.

Throughout the patient's hospital course, her head and neck pain were poorly controlled on both oral and intravenous analgesic medications. She described her headache as throbbing and circumferential, with increased intensity in the left frontal region. Headache was associated with severe posterior neck pain radiating to the upper back. She rated her headache pain at a level of 10 out of 10 using a 10-point numerical pain scale (NPS). Initially, she was started on scheduled acetaminophen-codeine $600-60 \mathrm{mg}$ every $6 \mathrm{hrs}$, naproxen $500 \mathrm{mg}$ twice daily, and tramadol 
$100 \mathrm{mg}$ every $6 \mathrm{hrs}$; this regimen provided minimal relief for her pain. Immediate-release oral morphine $15 \mathrm{mg}$ every $12 \mathrm{hrs}$ and pregabalin $50 \mathrm{mg}$ every $8 \mathrm{hrs}$ were added without any benefit.

Acute Pain Management Service (APMS) was consulted because the primary service was unable to discharge the patient on hospital day seven due to uncontrolled pain. The patient initially endorsed severe headache and neck pain exacerbated by bright lights and sitting in an upright position with minimal relief from medications. Given that she had undergone LP 3 days prior to APMS evaluation, and the positional nature of the headache, a diagnosis of post-dural puncture headache (PDPH) was considered. However, she was not a candidate for an epidural blood patch (EBP) because of her meningitis. The patient was started on caffeine-acetaminophen 200-650 mg every $6 \mathrm{hrs}$, with oxycodone $5 \mathrm{mg}$ by mouth every 4 hrs as needed for breakthrough pain.

The patient's headache and cervical pain were not relieved with this regimen, so the decision was made to perform bilateral T4 ESP blocks as described above on hospital day number eight. There were no complications from the procedure. Thirty minutes after the block was completed, the patient reported that the neck pain had decreased from a level of 10 on the NPS to a 2, and that the headache decreased from a level of 7 on the NPS to 0 . She was able to sit upright and perform a valsalva and rotate her neck without exacerbation of the headache or cervical pain, which she had previously been unable to do. She also reported that neck stiffness was alleviated. All opioids were discontinued and she was discharged home the following day. She was contacted approximately 1 month after discharge and reported that her headache never returned.

\section{Case 2 - Headache Following Craniotomy for Pineal Cyst Resection}

An 18-year-old obese female with a body mass index of 33.4 and a history of irritable bowel syndrome (IBS) and severe gastroparesis presented to APMS during readmission for inability to tolerate oral intake following supracerebellar transtentorial resection of $10 \mathrm{~cm}$ pineal cyst. Patient's incision is shown in Figure 2. She had been discharged home on postoperative day (POD) two and readmitted for severe headache, nausea, vomiting, and constipation on POD three. The headache was primarily localized to the frontal region with associated photophobia. Neurologic exam was nonfocal, and imaging of the head revealed expected postoperative changes. She was started on acetazolamide $500 \mathrm{mg}$ BID, dexamethasone $10 \mathrm{mg}$ intravenously followed by $4 \mathrm{mg}$

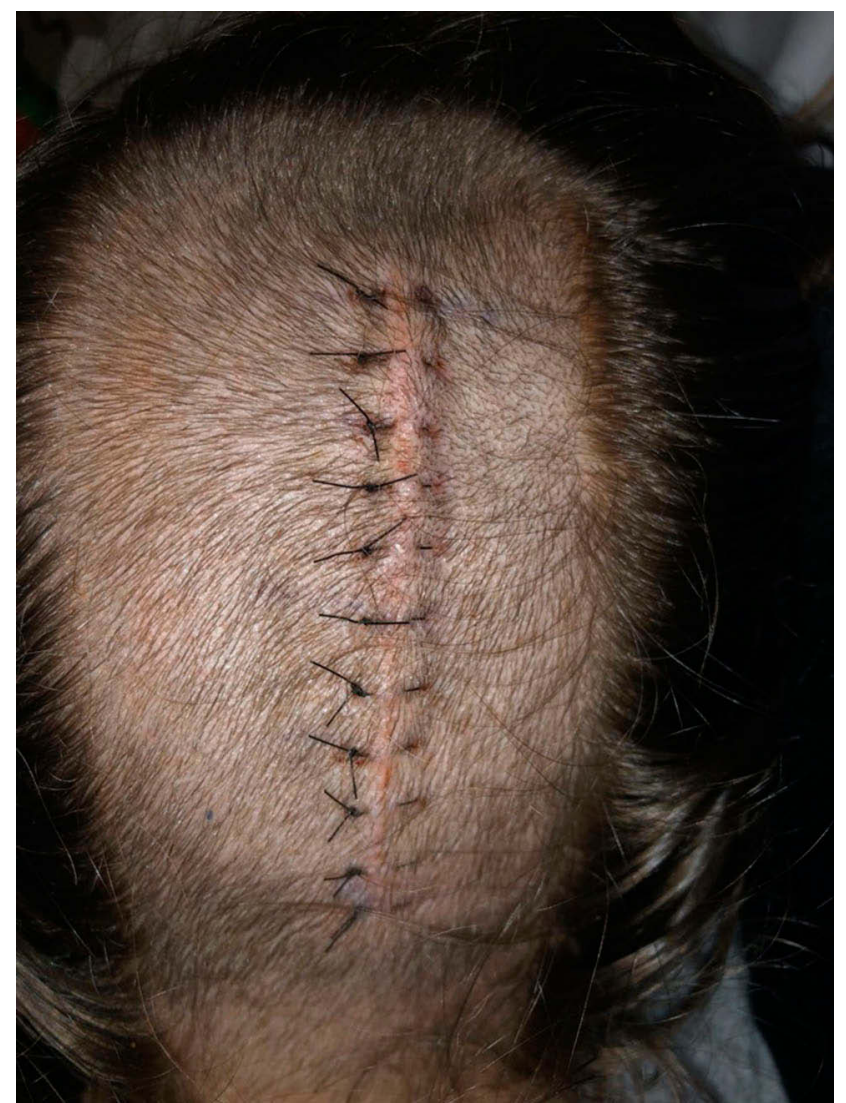

Figure 2 Pineal cyst resection - craniotomy incision.

every 6 hrs. LP was performed for assessment of opening pressure, which was found to be elevated at $29 \mathrm{~cm}$ of water pressure; profile of CSF obtained during the LP was not consistent with infection or aseptic meningitis. Pain was initially managed by the primary team with as-needed acetaminophen, acetaminophen-hydrocodone, ibuprofen, ketorolac, and fentanyl. APMS was consulted for narcotic-sparing pain control due to her severe gastroparesis, constipation, vomiting and IBS; she was started on a multimodal regimen of scheduled acetaminophen, gabapentin, ketorolac, and dronabinol, with tramadol 50 to $100 \mathrm{mg}$ every $4 \mathrm{hrs}$ as needed for breakthrough pain with the minimal improvement of pain score on NPS. Bilateral T4 ESP blocks were performed to augment the narcotic-sparing multimodal pain regimen. Within 10 mins following completion of the block, patient reported complete relief of headache. During follow-up evaluation the following day, the patient continued to rate her pain as a zero out of 10 on the VAS pain scale. During followup phone call 1 month after discharge, the patient reported that her headache recurred approximately 2 weeks following discharge, but never reached the level of her pain prior to the ESP block. 


\section{Case 3 - Chronic Headache and Scalp Pain After Craniotomy}

A 70-year-old male with a past medical history of peripheral artery disease, four coronary artery stents, two prior arterial ischemic strokes with residual right-sided weakness, anterior communicating artery aneurysm with several failed endovascular coilings, intracerebral hemorrhage, and subsequent right craniotomy for open aneurysm clipping 4 years prior to admission presented with a severe headache. He endorsed chronic moderate headache, scalp, and facial pain that was being managed by both a neurologist and a chronic pain specialist on an outpatient basis. He had been prescribed several different medical regimens including antidepressants, muscle relaxants, anticonvulsants, and narcotics without adequate pain relief. He had also undergone several trigger-point injections which failed to provide pain control. He was admitted due to an increase in severity of headache with a new onset of nausea.

Upon evaluation by APMS, his headache was throbbing in nature, primarily localized to the frontal and occipital regions. His scalp pain was sharp and radiated from the incisional scar on the right side of his head caudally along the right side of his face and neck; it fluctuated in severity from a five to 10 on a 10-point pain NPS. He was evaluated by the neurology service and placed on a scheduled regimen of acetaminophen $650 \mathrm{mg}$ by mouth every $6 \mathrm{hrs}$, tramadol $50 \mathrm{mg}$ PO every $8 \mathrm{hrs}$, as well as as-needed cyclobenzaprine $10 \mathrm{mg}$ every $8 \mathrm{hrs}$, and one-time intravenous doses of methylprednisolone $1 \mathrm{mg}$, morphine $4 \mathrm{mg}$, and zofran $4 \mathrm{mg}$. Despite this regimen, he continued to have uncontrolled head and neck pain with nausea.

Due to the failure of initial pain management, the neurology team consulted APMS for further assistance. At the time of consultation, the patient reported 10 out of 10 right-sided facial pain, neck pain and headache. Bilateral T4 ESP blocks were performed as previously described without complications. The patient reported improved pain from his headache within 15 mins of completion of the block. A follow-up evaluation was performed roughly $1 \mathrm{hr}$ following the procedure, and the patient continued to report complete alleviation of his headache, along with scalp and neck pain.

\section{Case 4 - Acute on Chronic Headache Following Craniotomy}

A 58-year-old male who had undergone resection of a brainstem cavernous sinus arteriovenous malformation 3 months prior to admission, but had recurrent bouts of aseptic meningitis resulting in headache. This headache was responsive to steroids and oral hydrocodone-acetaminophen 10/ $325 \mathrm{mg}$ every 4 to $6 \mathrm{hrs}$. It was believed that this was likely due to an allergic reaction to the synthetic dural patch used during closure, so he was readmitted for removal of the synthetic dural patch and replacement with native graft from his fascia lata. Immediately postoperatively he endorsed a pain score of 10 on the NPS; he characterized his headache as occipital and frontal in location, with an extension down the posterior aspect of his neck. His post-operative headache and incisional pain were unresponsive to intravenous and oral medications, including acetaminophen $1000 \mathrm{mg}$, hydromorphone $3 \mathrm{mg}$, fentanyl $350 \mathrm{mcg}$, and ketamine $50 \mathrm{mg}$, oxycodone $5 \mathrm{mg}$, gabapentin $300 \mathrm{mg}$, and methocarbamol $1000 \mathrm{mg}$. Due to his uncontrolled pain, he was unable to be transferred from the post-anesthesia care unit to the neurosurgical unit. APMS was consulted for intractable headache, and performed bilateral ultrasound-guided T4 ESP blocks as previously described. Fifteen minutes following the block he endorsed a reduction in his pain score from 10 to 2 . He was monitored for 30 mins following completion of the block and was then transferred to the neurosurgery unit. On postoperative day 1 , the patient reported improved functional status and mobility, with a headache pain score of four.

\section{Discussion}

Cervical and high thoracic ESP has been described at multiple spinal levels including at $\mathrm{C} 7$ for shoulder disarticulation surgery, T2 and T3 for chronic shoulder pain, axillary pain, and cervical spine surgery, as well as at $\mathrm{T} 4$ for tension headaches and carotid endarterectomy surgery. ${ }^{17-22}$ Although the ESP block is currently primarily used for acute thoracoabdominal pain, it may also be a reasonable adjunct therapy when conventional treatment for headache and cervical pain fail. We reported four cases in which bilateral T4 ESP blocks provided significant relief of head and neck pain and secondary headache.

Peripheral nerve blocks and ganglion blocks are established treatments for primary and some secondary headaches. GON blocks have been used to treat migraine, occipital neuralgia, cluster, cervicogenic, post-concussive, and postdural puncture headaches. ${ }^{23-29}$ SPG block has also been used to relieve headaches, including migraine, cluster and ${ }^{\text {PDPH. }}{ }^{29-33}$ Stellate ganglion blockade was studied in the treatment of posttraumatic headache. ${ }^{34}$ ESP blocks have demonstrated effectiveness in relieving tension headaches, a type of primary headache. ${ }^{17}$ This is the first case series 
reporting successful treatment of secondary headaches with ESP blocks.

For the patient in case one, we opted not to utilize an SPG block since the patient reported greater pain scores in her neck than her head, and we hypothesized that the ESP block would more adequately cover the two regions than the SPG block. In case two, the decision to perform ESP blocks was made due to the location of the surgical incision (see Figure 1), which limited access for GON and LON blocks. The patient in case three presented with postcraniotomy head and neck pain with no dermatomal pattern, which was refractory to medical management for almost 4 years. ESP block was performed given the dual location of pain in his head and neck. The patient in case four also endorsed pain in the head and neck which we believed would be amenable to ESP blockade.

Most regional anesthetic techniques used to relieve headaches are simple to perform and involve small amounts of local anesthetic. ${ }^{35}$ The transnasal SPG block can be performed with an intranasal catheter or by inserting a local anesthetic soaked cotton-tipped applicator above the middle turbinate until contact is made with the posterior nasopharynx. GON and LON blocks can be performed with landmark technique or ultrasound-guidance and require less than $5 \mathrm{~mL}$ of local anesthetic per side. Supraorbital, supratrochlear, and auriculotemporal nerve blocks are landmark-based and are performed with one or two $\mathrm{mL}$ of local anesthetic. However, high thoracic ESP blocks require ultrasound-guidance to ensure proper injection of a large volume of local anesthetic, usually $20 \mathrm{~mL}$ per side, into the correct fascial plane between muscles, as intramuscular injection of local anesthetic will result in block failure. When injecting high doses of cardiotoxic local anesthetics, it is recommended to use standard American Society of Anesthesiologists monitors and have appropriate resuscitation medications (ie lipid emulsion) and intubation equipment readily available. Despite the ESP block being perceived as technically simple, pneumothorax has been reported. $^{36,37}$ Two alternative scanning and needling approaches have been proposed to prevent pneumothorax during ESP block. Aksu et al recommend placing the ultrasound probe in the transverse position and inserting the needle out-of-plane; however, the pleura can still be violated with this technique. ${ }^{38}$ To prevent inadvertent pleural puncture, an inplane approach visualizing the needle tip at all times is preferred as described by Piraccini et al. ${ }^{39}$ With cervical and high thoracic ESP block, there is a concern for phrenic nerve blockade and associated respiratory distress. Because we performed bilateral ESP, we chose high thoracic over cervical
ESP. There are far more reports of T4 ESP than cervical ESP. Phrenic nerve blockade after high thoracic ESP has been examined in a study by Ueshima et al, and despite visualization of the spread of local anesthetic to the cervical nerve roots, hemidiaphragmatic paresis has not been shown. The investigators retrospectively analyzed the rate of hemidiaphragmatic paresis after T4 ESP block for carotid endarterectomy: the study concluded that high thoracic ESP block did not anesthetize the anterior branches of the cervical spinal nerves or phrenic nerve. ${ }^{40}$ Paravertebral spread of local anesthetic is likely after ESP block as evidenced by the following complications: Harlequin syndrome, a self-limited partial autonomic neuropathy after a T3 ESP block, and an unexpected bilateral sensory deficit after T9 ESP block have been reported and attributed to paravertebral spread. ${ }^{41,42}$ Thus, patients should not be considered as candidates for high thoracic ESP if they would not tolerate a high thoracic epidural or paravertebral block. The American Society of Regional Anesthesia (ASRA) Guidelines recommend avoiding deep peripheral nerve blocks in anticoagulated patients. ${ }^{43}$ Although concerns regarding the risks of epidural hematoma after ESP block have been raised, there are no major neurovascular structures in the area of injection and are no reported cases of ESP block causing clinically significant bleeding. ${ }^{44}$ Local anesthetic spread into the paravertebral space may occur due to high pressures achieved during manual injection with a syringe, however, should vascular injury occur during the ESP block, it is unlikely that bleeding from a vessel would achieve the same level of pressure, and spread into the epidural space. ESP block should be performed by a physician experienced with ultrasound-guided regional anesthesia techniques, and management of possible complications.

Interventional headache management includes triggerpoint injections, peripheral nerve stimulators, as well as peripheral nerve and ganglion blocks using steroids, local anesthetics and other adjuvants. ESP block is generally performed using long-acting local anesthetics with or without steroids. It has been suggested that local anesthetics break the headache cycle through central pain modulation at the level of the spinal cord by lowering peripheral afferent excitability and blocking synaptic transmission. ${ }^{45}$ Another proposed mechanism of action is autonomic blockade including both sympathetic and parasympathetic nuclei which leads to control of intracranial vasomotor function. Local anesthetics also have significant anti-inflammatory properties, blocking the release of cytokines and interfering with the migration of inflammatory cells. ${ }^{46}$ We believe the local anesthetic injected in a T4 ESP blocks the upper 
cervical nerve roots via the trigeminocervical complex disrupting central sensitization implicated in headaches, meningeal inflammation and referred pain. ${ }^{8}$ Despite the varying etiology of secondary headaches, it is possible that the end result is activation and excitation of the autonomic system and trigeminocervical complex, and thus treatment with local anesthetic injected in an ESP block ameliorates headache pain as described above.

\section{Conclusion}

When medical management is inadequate, several interventions have been described for the treatment of headache, especially primary headaches. The ESP block may be an additional tool in the treatment of medically refractory secondary headaches and should be considered over other peripheral nerve blocks in patients with concomitant neck pain in addition to severe headache. There is growing literature for the efficacy of ESP block and refractory or intractable secondary headache management is another potential indication.

\section{Acknowledgments}

The authors would like to acknowledge the Memorial Hermann Health System.

\section{Disclosure}

The authors report no conflicts of interest in this work.

\section{References}

1. Schulman EA, Lee Peterlin B, Lake AE, et al. Defining refractory migraine: results of the RHSIS survey of American headache society members. Headache. 2009;49(4):509-518. doi:10.1111/j.15264610.2009.01370.x

2. Arnold M. Headache classification committee of the International Headache Society (IHS) the international classification of headache disorders, 3rd edition. Cephalalgia. 2018;38(1):1-211. doi:10.1177/ 0333102417738202

3. Blumenfeld A, Ashkenazi A, Napchan U, et al. Expert consensus recommendations for the performance of peripheral nerve blocks for headaches-a narrative review. Headache. 2013;53(3):437-446. doi:10.1111/head.12053

4. Blumenfeld A, Ashkenazi A. Nerve blocks, trigger point injections and headache. Headache. 2010;50(6):953-954. doi:10.1111/j.15264610.2010.01685.x

5. Naja ZM, El-Rajab M, Al-Tannir MA, et al. Occipital nerve blockade for cervicogenic headache: a double-blind randomized controlled clinical trial. Pain Pract. 2006;6(2):89-95. doi:10.1111/j.1533-2500. 2006.00068.x

6. Naja Z, Al-Tannir M, El-Rajab M, et al. Nerve stimulator-guided occipital nerve blockade for postdural puncture headache. Pain Pract. 2009;9(1):51-58. doi:10.1111/j.1533-2500.2008.00238.x

7. Niraj G, Kelkar A, Girotra V. Greater occipital nerve block for postdural puncture headache $(\mathrm{PDPH})$ : a prospective audit of a modified guideline for the management of PDPH and review of the literature. J Clin Anesth. 2014;26(7):539-544. doi:10.1016/j.jclinane.2014. 03.006
8. De Haan JB, Chrisman OM, Linden Lee MG, Hernandez N. T4 erector spinae plane block relieves postdural puncture headache: a case report. Cureus. 2019;11(11):e6237.

9. Forero M, Adhikary SD, Lopez H, et al. The erector spinae plane block: a novel analgesic technique in thoracic neuropathic pain. Reg Anesth Pain Med. 2016;41(5):621-627. doi:10.1097/AAP.0000000000000451

10. Evans HT, Leslie GJ, Rutka O, et al. Bilateral erector spinae plane block for surgery on the posterior aspect of the neck: a case report. A a Pract. 2019;12(10):356-358. doi:10.1213/XAA.00000000000000926

11. Brandao J, Graça R, Sá M, et al. Lumbar erector spinae plane block: successful control of acute pain after lumbar spine surgery - a clinical report. Rev Esp Anestesiol Reanim. 2019;66(3):167-171. doi:10. 1016/j.redar.2018.10.005

12. Tsui BCH, Ip VHY. Can electrical nerve stimulation guidance assist in cervical erector spinae plane block catheter placement for total shoulder arthroplasty? Can J Anaesth. 2019;66(11):1417-1418. doi:10.1007/s12630-019-01457-x

13. Chin KJ, Malhas L, Perlas A. The erector spinae plane block provides visceral abdominal analgesia in bariatric surgery: a report of 3 cases. Reg Anesth Pain Med. 2017;42(3):372-376. doi:10.1097/AAP.00 00000000000581

14. Rincon C, Moreno DA, Moore A. Erector spinae plane block for post-cesarean delivery analgesia. Int J Obstet Anesth. 2019.

15. Yao Y, Li H, He Q, Chen T, Wang Y, Zheng X. Efficacy of ultrasound-guided erector spinae plane block on postoperative quality of recovery and analgesia after modified radical mastectomy: randomized controlled trial. Reg Anesth Pain Med. 2019.

16. Ayub A, Talawar P, Gupta SK, Kumar R, Alam A. Erector spinae plane block: a safe, simple and effective alternative for knee surgery. Anaesth Intensive Care. 2019;310057X19877655.

17. Ueshima H, Otake H. Successful cases of bilateral erector spinae plane block for treatment of tension headache. J Clin Anesth. 2019;54:153. doi:10.1016/j.jclinane.2018.12.009

18. Hamadnalla H, Elsharkawy H, Shimada T, et al. Cervical erector spinae plane block catheter for shoulder disarticulation surgery. Can J Anaesth. 2019;66(9):1129-1131. doi:10.1007/s12630-019-01421-9

19. de Haan J, Hernandez N, Sen S. Erector spinae block for postoperative analgesia following axillary hidradenitis suppurative resection: a case report. Local Reg Anesth. 2018;11:87-90. doi:10.2147/LRA. S179830

20. Forero M, Rajarathinam M, Adhikary SD, et al. Erector spinae plane block for the management of chronic shoulder pain: a case report. Can J Anaesth. 2018;65(3):288-293. doi:10.1007/s12630-017-10101

21. Ueshima H, Otake H. Blocking of multiple posterior branches of cervical nerves using an erector spinae plane block. J Clin Anesth. 2018;46:44. doi:10.1016/j.jclinane.2018.01.021

22. Ueshima H, Hiroshi O. Erector spinae plane block for carotid endarterectomy. $J$ Clin Anesth. 2018;48:11. doi:10.1016/j. jclinane.2018.04.004

23. Afridi SK, Shields KG, Bhola R, et al. Greater occipital nerve injection in primary headache syndromes-prolonged effects from a single injection. Pain. 2006;122(1-2):126-129. doi:10.1016/j.pain.2006.01.016

24. Ashkenazi A, Matro R, Shaw JW, et al. Greater occipital nerve block using local anaesthetics alone or with triamcinolone for transformed migraine: a randomised comparative study. J Neurol Neurosurg Psychiatry. 2008;79(4):415-417. doi:10.1136/jnnp.2007.124420

25. Young W, Cook B, Malik S, et al. The first 5 minutes after greater occipital nerve block. Headache. 2008;48(7):1126-1128. doi:10.1111/j.1526-4610.2008.01146.x

26. Anthony M. Headache and the greater occipital nerve. Clin Neurol Neurosurg. 1992;94(4):297-301. doi:10.1016/0303-8467(92)90177-5

27. Hecht JS. Occipital nerve blocks in postconcussive headaches: a retrospective review and report of ten patients. J Head Trauma Rehabil. 2004;19(1):58-71. doi:10.1097/00001199-20040100000006 
28. Matute E, Bonilla S, Gironés A, et al. Bilateral greater occipital nerve block for post-dural puncture headache. Anaesthesia. 2008;63 (5):557-558. doi:10.1111/j.1365-2044.2008.05531.x

29. Costa A, Pucci E, Antonaci F, et al. The effect of intranasal cocaine and lidocaine on nitroglycerin-induced attacks in cluster headache Cephalalgia. 2000;20(2):85-91. doi:10.1046/j.1468-2982.2000.00026.x

30. Binfalah M, Alghawi E, Shosha E, Alhilly A, Bakhiet M. Sphenopalatine ganglion block for the treatment of acute migraine headache. Pain Res Treat. 2018;2018:2516953.

31. Hardebo JE, Elner A. Nerves and vessels in the pterygopalatine fossa and symptoms of cluster headache. Headache. 1987;27(10):528-532. doi:10.1111/j.1526-4610.1987.hed2710528.x

32. Kittrelle JP, Grouse DS, Seybold ME. Cluster headache. Local anesthetic abortive agents. Arch Neurol. 1985;42(5):496-498. doi:10. 1001/archneur.1985.04060050098017

33. Singla D, Mangla M. Sphenopalatine ganglion block: a newer modality for management of postdural puncture headache. J Anaesthesiol Clin Pharmacol. 2018;34(4):567-568. doi:10.4103/joacp.JOACP_64_17

34. Chan CW, Chalkiadis GA. A case of sympathetically mediated headache treated with stellate ganglion blockade. Pain Med. 2010;11 (8):1294-1298. doi:10.1111/j.1526-4637.2010.00850.x

35. Levin M. Nerve blocks in the treatment of headache. Neurotherapeutics. 2010;7(2):197-203. doi:10.1016/j.nurt.2010.03.001

36. Ueshima H. Pneumothorax after the erector spinae plane block. J Clin Anesth. 2018;48:12. doi:10.1016/j.jclinane.2018.04.009

37. Hamilton DL. Reducing the risk of pneumothorax following erector spinae plane block. J Clin Anesth. 2019;56:3. doi:10.1016/j. jclinane.2019.01.004

38. Aksu C, Gurkan Y. Erector spinae plane block: a new indication with a new approach and a recommendation to reduce the risk of pneumothorax. J Clin Anesth. 2019;54:130-131. doi:10.1016/j. jclinane.2018.11.007
39. Piraccini E, Poggi P, Maitan S. An alternative approach to reduce the risk of pneumothorax during erector spinae plane block in low thoracic vertebral levels. $J$ Clin Anesth. 2020;59:14-15. doi:10.1016/j.jclinane.2019.06.005

40. Ueshima H, Otake H. The influence of phrenic nerve paralysis for a thoracic erector spinae plane block. $J$ Clin Anesth. 2019;55:113-114. doi:10.1016/j.jclinane.2018.12.021

41. Sullivan TR, Kanda P, Gagne S, et al. Harlequin syndrome associated with erector spinae plane block. Anesthesiology. 2019;131(3):665. doi:10.1097/ALN.0000000000002733

42. Tulgar S, Selvi O, Ahiskalioglu A, et al. Can unilateral erector spinae plane block result in bilateral sensory blockade? Can J Anaesth. 2019;66(8):1001-1002. doi:10.1007/s12630-019-01402-y

43. Horlocker TT, Vandermeuelen E, Kopp SL, et al. Regional anesthesia in the patient receiving antithrombotic or thrombolytic therapy: American society of regional anesthesia and pain medicine evidence-based guidelines (fourth edition). Reg Anesth Pain Med. 2018;43(3):263-309. doi:10.1097/AAP.0000000000000763

44. Smith CA, Martin KM. Dual antiplatelet therapy does not scare away the erector spinae plane block. Korean J Anesthesiol. 2019;72 (3):277-278. doi:10.4097/kja.19044

45. Moldovan M, Lange KHW, Aachmann-Andersen NJ, et al. Transient impairment of the axolemma following regional anaesthesia by lidocaine in humans. J Physiol. 2014;592(13):2735-2750. doi:10.1113/ jphysiol.2014.270827

46. Estebe JP. Intravenous lidocaine. Best Pract Res Clin Anaesthesiol. 2017;31(4):513-521. doi:10.1016/j.bpa.2017.05.005
Local and Regional Anesthesia

\section{Publish your work in this journal}

Local and Regional Anesthesia is an international, peer-reviewed, open access journal publishing on the development, pharmacology, delivery and targeting and clinical use of local and regional anesthetics and analgesics. The journal welcomes submitted papers covering original research, basic science, clinical studies, reviews \&

\section{Dovepress}

evaluations, guidelines, expert opinion and commentary, case reports and extended reports. The manuscript management system is completely online and includes a very quick and fair peer-review system, which is all easy to use. Visit http://www.dovepress.com/testimonials. php to read real quotes from published authors. 